Published in final edited form as:

Curr Opin Lipidol. 2010 June ; 21(3): 212-217.

\title{
Acyl-coenzyme A synthetases in metabolic control
}

\author{
Jessica M. Ellis, Jennifer L. Frahm, Lei O. Li, and Rosalind A. Coleman \\ Department of Nutrition, University of North Carolina, Chapel Hill, North Carolina, USA
}

\section{Abstract}

Purpose of review-The 11 long-chain (ACSL) and very long chain acyl-coenzyme A (acyl$\mathrm{CoA})$ synthetases [(ACSVL)/fatty acid transport protein] are receiving considerable attention because it has become apparent that their individual functions are not redundant.

Recent findings-Recent studies have focused on the structure of the acyl-CoA synthetases, their post-translational modification, their ability to activate fatty acids of varying chain lengths, and their role in directing fatty acids into different metabolic pathways. An unsettled controversy focuses on the ACSVL isoforms and whether these have both enzymatic and transport functions. Another issue is whether conversion of a fatty acid to an acyl-CoA produces an increase in the AMP/ATP ratio that is sufficient to activate AMP-activated kinase.

Summary-FuturestudiesarerequiredtodeterminethesubcellularlocationofeachACSLandACSVL isoform and the functional importance of phosphorylation and acetylation. Purification and crystallization of mammalian ACSL and ACSVL isoforms is needed to confirm the mechanism of action and discover how these enzymesdiffer in their affinity for fatty acids of differentchainlengths.Functionally, itwillbeimportanttolearnhowtheACSLisoformscan direct their acyl-CoA products toward independent downstream pathways.

\section{Keywords}

$\beta$-oxidation; acyl-CoA synthetase; AMP-activated kinase; fatty acid; fatty acid transport protein; glycerolipid synthesis

\section{Introduction}

Of the 26 mammalian acyl-coenzyme A (acyl-CoA) synthetases (ACS), at least 11 can activate the major long-chain fatty acids (FAs) of $16-22$ carbons $\left[1,2^{\circ}\right]$. These include members of the long-chain [long-chain acyl-CoA synthetases (ACSL)] and very long chain [very long chain acyl-CoA synthetases (ACSVL)/fatty acid transport protein (FATP)] subfamilies that catalyze a two-step reaction:

$$
\begin{aligned}
& F A+A T P \rightarrow a c y l-A M P+P P i \text { (pyrophosphate) } \\
& A c y l-A M P+\text { Coenzyme } \quad \mathrm{A} \rightarrow a c y l-C o A+A M P
\end{aligned}
$$


This review will focus primarily on very recent advances in the study of these long-chain ACSs in metabolic regulation, highlighting new studies on structure, function, and regulation.

\section{Structure and sequence of acyl-coenzyme A synthetase}

Acyl-CoA synthetases are homologous in their sequences. The ACS catalytic domain shares homology with the ANL (A Acyl-CoA synthetases, Nonribosomal peptide synthetase adenylation domains, and Luciferase enzymes) super-family, a family consisting of ACS, nonribosomal peptide synthetase, and luciferase [ $3^{\circ *}$. The homology between ACS and luciferase is so similar that mutation of a single residue (L345S) allows the Drosophilia melangaster ACS to gain luciferase activity [ $4^{{ }^{*}}$ ]. For all ANL superfamily enzymes, the Cterminal domain is rotated $\sim 140^{\circ}$ after adenylation-formation; in ACS this rotation results in the conformation that forms thioesters. Analysis of the only reported mammalian ACS crystal structure revealed the following: (1) in the adenylate-forming conformation a conserved lysine, $\mathrm{Mg}^{2+}$, and the P-loop holds the pyro-phosphate in the active site; (2) in the thioester conformation the Arg472-Glu365 salt bridge sterically blocks ATP binding; and (3) the switch of the adenylate-forming conformation to the thioesterase conformation requires the presence of pyrophosphate $\left[5^{\circ}\right]$.

\section{Acyl-coenzyme A synthetase and fatty acid uptake}

FA may enter cells via both concentration-based flipping and protein-mediated transport [6]. Cellular retention may depend on 'vectorial acylation', the trapping of exogenous FA as an acyl-CoA within the cell and its subsequent use in downstream metabolism [7]. Although FATP1 was discovered in a functional FA uptake screen [8], several FATP family members are not present on the plasma membrane [7], and so they are also called ACSVL to distinguish their ability to activate FA with chain lengths as long as 26 carbons [1].

Although FA uptake increases in NIH fibroblasts [9] and COS ( $\underline{\mathrm{CV}}-1$ (simian) in $\underline{\text { Origin, and }}$ carrying the $\underline{S} V 40$ genetic material) cells overexpressing ACSL1 [10], it is unaffected by over-expressing [11] or knocking out ACSL1 in primary hepatocytes [12**] or 3T3-L1 adipocytes $\left[13^{* *}\right]$. The conflicting data concerning results of FA uptake by the same ACSL isoforms likely reflect differences in techniques for measuring uptake [7], the artificial effect of overexpression, tissue-specific subcellular location, and tissue-specific interacting proteins.

\section{Fatty acid channeling}

Although each ACSL and ACSVL isoform activates a broad range of long-chain FA, their respective physiological roles remain unknown. We hypothesized that different ACS isoforms channel FA into specific downstream pathways that could vary in a tissue-specific manner [7]. For example, despite a 50\% decrease in total ACSL activity, primary hepatocytes from ACSL1 liver-specific knockout mice had small reductions in FA incorporation into both triacylglycerol (TAG) and $\beta$-oxidation products [12*0. The ACSL1 knockout livers had reduced long-chain acyl-carnitine content and reduced rates of FA oxidation. The minimal phenotype suggests that alternative pathways for FA oxidation and TAG synthesis are active in the $A c s l^{L-/-}$ liver. 
In contrast, primary epididymal adipocytes from ACSL1 adipose-specific knockout mice have unchanged FA incorporation into TAG, diacylglycerol (DAG), or phospholipid (PL), but reduced $\beta$-oxidation products (J.M. Ellis, R.A. Coleman, unpublished data). However, siRNA knockdown of ACSL1 in 3T3-L1 adipocytes does not affect de-novo lipogenesis or FA oxidation, but increases FA efflux [13**], leading the authors to conclude that ACSL1 activates FA that is re-esterified after lipolysis. Pulse-chase labeling experiments could verify reduced rates of re-esterification and confirm this interpretation.

Knocking down ACSL3 in rat primary hepatocytes decreases labeled acetate incorporation into TAG and PL [14*0]. In human hepatoma Huh7 cells, siRNA knockdown of ACSL3 decreases oleate incorporation into phosphatidylcholine (PC) and secreted VLDL and increases cellular-free oleate, whereas knockdown of ACSL1 and ACSL4 does not alter oleate incorporation into cholesterol ester (CE), TAG, phospholipids, or free FA [15**]. Differential effects of individual ACSL iso-forms support the notion that ACSL isoforms channel FA to distinct metabolic fates.

Several overexpression studies are inconsistent with the knockdown studies and provide strikingly contradictory interpretations of the role of ACSL1. For example, when ACSL1 is overexpressed in rat primary hepatocytes, FAs are incorporated into DAG and phospholipids but not into CEorsecretedTAG[11].OverexpressedACSL5increases FA incorporation into DAG and TAG but does not affect FA used for $\beta$-oxidation [16]. In contrast, however, overexpressing either human ACSL3 or rat ACSL5 in HepG2 cells increases palmitate oxidation [17]. Further, TAG content increases in NIH-3T3 fibroblasts when ACSL1/ FATP1 is overexpressed [18] and in insulin-stimulated 3T3-L1 adipocytes when ACSL1 is knocked down $\left[13^{*}\right]$.

We see two potential problems. The first is that over-expression of an enzyme may overwhelm downstream pathways that cannot readily accommodate excess substrate; the second problem is that studies in cell lines are not always translatable to primary cells. Thus, proteomic analysis of mitochondria isolated from white adipose tissue, brown adipose tissue, and 3T3-L1 adipocytes reveals substantial differences [19*0. Compared with mitochondria from adipose tissue, many proteins are downregulated in 3T3-L1 mitochondria. Further, medium-chain ACS (ACSM) isoforms are more abundant than ACSL isoforms in white adipose tissue mitochondria, suggesting greater medium-chain FA oxidative metabolism. ACSL1 and ACSL6 are particularly enriched in white adipose mitochondria, whereas ACSL5 is more abundant in mitochondria from brown adipose. However, despite the high abundance of ACSL5 in brown adipose mitochondria, ACSL1 is required for adaptive thermo-genesis and normal FA oxidation in brown adipose (J.M. Ellis, R.A. Coleman, unpublished data). Thus, the function of ACSL5 in BAT remains unknown.

FA channeling could occur via protein-protein interactions. Immunoprecipitation of endogenous FATP1 from 3T3-L1 adipocytes followed by mass spectrometry identified mitochondrial 2-oxoglutarate dehydrogenase (OGDH), a key enzyme in the tricarboxylic acid cycle [20“]. FATP1 enhances OGDH activity in proteoliposomes, whereas FATP1 knockdown in 3T3-L1 adipocytes showed decreased OGDH and TCA cycle activity. Overexpressed FATP1 and carnitine palmitoyltransferase-1 (CPT1) also co-immunoprecipitate 
from L6E9 myotubes and rat skeletal muscle in vivo [21 ${ }^{\circ}$ ]. In L6E9 myotubes, FATP1 overexpression enhances FA oxidation, suggesting that protein-protein interactions of FATP1 facilitate activities of FA oxidation proteins. Overexpressed ACSL1 and FATP1 coimmunoprecipitate in 3T3-L1 adipocytes [22], but it is not known whether endogenous ACSL1 and FATP1 proteins interact.

The subcellular location of ACS enzymes, which was recently reviewed [23"], may influence the downstream metabolism of the acyl-CoA product. However, sub-cellular location studies have yielded conflicting interpretations. Most have examined subcellular fractions that are limited in purity. Data from overexpression studies are also problematic because the location of over-expressed proteins may not mimic the endogenous condition. In one such example, the subcellular locations of overexpressed and endogenous FATP4/ ACSVL5 differed [24]. Confocal microscopy with tested isoformspecific antibodies will be required to determine precise locations of the acyl-CoA synthetases.

\section{Acyl-coenzyme A synthetase enzyme regulation}

Broad mass spectrometry surveys reveal a number of post-translational modifications to ACS enzymes, although their functional significance is generally unknown. An exception is the regulation on Lys-609 located on the C-terminus of the Salmonella enterica, ACS, for which lysine acetylation inhibits activity by preventing formation of the thioester [25]. Similar regulation occurs by the sirtuin (SIRT) acetylases on Lys-661 and Lys-635 of acetylCoA synthetase- 1 and acetyl-CoA synthetase-2, respectively, and human acetyl-CoA synthetase 2 is regulated on Lys-642 [26]. In response to a calorie-restricted diet, acetylation of Lys-534 of ACSM1 and Lys-510 of acyl-CoA synthetase family member-2 (Q8VCW8) increased 2.75-fold and 2.3-fold, respectively [27*0].

\section{Role of acyl-coenzyme A synthetase in transcriptional regulation}

ACSL can theoretically regulate transcription by modulating the intracellular content of FA and acyl-CoA, which are ligands for transcription factors. FA, for example, is a ligand for peroxisome proliferator-activated receptor (PPAR) a, $\beta$, or $\delta$ and may suppress SREBP1c target genes, and acyl-CoA is a possible ligand for HNF4a [28]. The role of ACS in regulating transcription was addressed by a study that used siRNA to knock down the longchain ACS isoforms that are expressed in liver, ACSL1, 3, 4, and 5, and FATP2, 4, and 5 [14*0]. In primary rat hepatocytes, only an siRNA that targeted ACSL3 had effects on transcription. This siRNA elevated intracellular FA $82 \%$ and diminished the expression of PPAR $\gamma$, as well as reporter gene activity for PPAR $\gamma$, ChREBP, SREBP1c, and liver X receptor a (LXRa), together with the expression of target genes for these transcription factors. Decreased use of $\left[1-{ }^{14} \mathrm{C}\right]$ acetate for de-novo lipogenesis and complex lipid synthesis confirmed the effect of diminishing the expression of genes involved in lipid synthesis. Although one might conclude that the knockdown of ACSL3 decreased these transcriptional activities via an increase in a specific pool of FA ligand, $\mathrm{Bu}$ et al. argue that ACSL3 must control transcription in a ligand-independent manner because synthetic ligands did not normalize transcriptional activities of LXR and PPAR $\gamma$. 


\section{Long-chain acyl-coenzyme A synthetase's role in disease}

ACSL is required for complex lipid synthesis in rapidly growing cells and the fungal metabolite triacsin C was initially reported to inhibit ACSL, diminish the synthesis of phospholipids, and decrease cell proliferation [29]. Triacsin C caused apoptosis in glioma cells, and, although it inhibits ACSL1, 3, and 4, but not ACSL5, overexpression of ACSL5 rescued the glioma cells $\left[30^{\circ}, 31^{\circ}\right]$. In this case, ACSL5 appeared to compensate for the inhibited isoforms. In a related publication, ACSVL3 (FATP3) was elevated in human gliomas [ $\left.32^{\circ}\right]$. ACSVL3 siRNA inhibited glioma cell growth and the cells became less tumorigenic. Because these features were reversed when active myr-Akt was expressed, ACSVL3 was thought to maintain oncogenesis by regulating Akt function.

Mutations in ACS genes have been identified in large screening studies. An Acsl5 single nucleotide polymorphism (SNP) (rs2419621; C >T) increased Acsl5 mRNA abundance in skeletal muscle and was associated with improved weight loss. This SNP increases the transcriptional rate of Acsl5 by enhancing gene activation by MyoD [33 ${ }^{\circ}$. Because this ACSL5 SNP increases the differentiation of skeletal myoblast cells, persons with this mutation may have an advantageous ability to increase muscle mass and caloric expenditure with diet and exercise.

High levels of Acsl3 gene methylation in umbilical cord white blood cells were strongly correlated with levels of maternal polycyclic aromatic hydrocarbons and with the mRNA

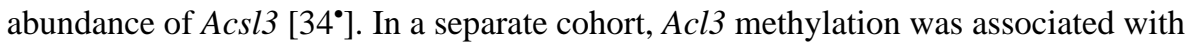

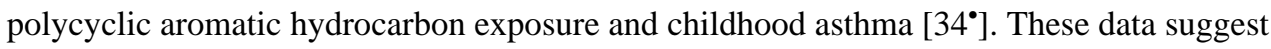
that maternal environmental exposures may affect childhood asthma through the methylation of the $\mathrm{Acl} 3$ gene.

The knockdown of Acsl4 in neurites reduces dendritic spine formation, suggesting a role for ACSL4 in the maturation and remodeling of neurons and providing a link to the X-linked mental retardation associated with human mutations in ACSL4 [35"0]. In another study, a SNP (rs1324805) in the Acsl4 gene was more prevalent in patients with the metabolic syndrome [36 ${ }^{\circ}$. A third study found an Acsl4 SNP (rs7887981) associated with increased liver fat or with increased circulating insulin and TAG in overweight participants from two different cohorts $\left[37^{\circ}\right]$. It is unknown how these SNPs affect the function of ACSL4, nor is it clear how the preference of ACSL4 for long-chain polyunsaturated FA may be involved.

A mutation within the Fatp4 gene is associated with the ichthyosis prematurity syndrome (IPS) [38 ${ }^{\circ}$. Fibroblasts from an IPS patient had reduced ACSVL activity and reduced incorporation rates of very long chain fatty acids into complex lipids. The skin findings are like those of Fatp4 null mice, which die in early life with hyper-proliferative hyperkeratosis [39], and suggest that FATP4 plays a role in early epidermal development and that other ACS enzymes cannot compensate for a deficit of this enzyme.

\section{Effect of fatty acid activation on AMP-activated kinase}

Because the synthesis of each acyl-CoA uses the energy equivalent of two ATP molecules and produces one AMP, it has been suggested that FA activation might increase cellular 
AMP/ATP ratios and activate AMP-activated kinase (AMPK) (Fig. 1). AMPK would then increase FA oxidation while blocking TAG synthesis. It would be surprising, however, if the conversion of FA to acyl-CoA triggered the activity of an energy-deficit indicator in settings of energy surplus when TAG synthesis is prominent.

Studies in animals have not been performed to compare ACSL-mediated activation of AMPK under different physiological conditions, but metabolic adaptations to fasting and chronic caloric restriction in heart, muscle, and liver do not include changes in AMPK activity [40], and when mice are placed in a cold environment, AMPK is not activated for several days despite increased ACSL-mediated FA activation [41].

Some experiments, however, do support ACSL-mediated activation of AMPK. In 3T3-L1 adipocytes, stimulation of lipolysis resulted in activated AMPK [42*0]. The authors suggested that activation of AMPK during lipolysis would 'restrain the energy depletion and oxidative stress'. It might also permit use of released FA for $\beta$-oxidation within the adipocyte and conserve glucose during a 'fast'. In a situation more akin to 'feeding', AMPK activation increases in 3T3-L1 adipocytes during insulin-stimulated palmitate uptake, although the AMP/ ATP ratio changes minimally [43**].

Studies in heart [44] and skeletal muscle $\left[45^{\circ}, 46,47\right]$ suggest that AMPK may be sensitive to the 'lipid status' of the cell and that activation may be influenced by intracellular FA availability, independent of cellular AMP levels, with FAs, themselves, activating AMPK $\left[48^{\circ}\right]$. FA added to L6 myotubes increases AMPK activity by 40-50\%, together with phosphorylation of AMPK Thr ${ }^{172}$ and acetyl-CoA carboxylase- $\beta \operatorname{Ser}^{218}$, and the rate of palmitate oxidation is almost doubled, without an increase in AMP content or LKB1/ AMPKK activation [49]. Dominant-negative AMPK adenovirus reduced basal FA oxidation and inhibited the stimulatory effects of FA pretreatment on FA oxidation, suggesting that FA might improve AMPK as a substrate for LKB1. However, in assays with purified components, 10-50 $\mu \mathrm{mol} / 1$ long-chain acyl-CoAs directly inhibited LKB1, suggesting that the rise in long-chain acyl-CoA content that occurs when FA synthesis is enhanced would negate the effect of increased AMP to prevent simultaneous synthesis and FA oxidation [50]. In addition, strong evidence supports the idea that protein kinase A phosphorylates and inactivates AMPK [51], thereby allowing hormone-sensitive lipase activation to diminish and slowing the rate of lipolysis. Although the cellular concentration of long-chain acylCoAs has been estimated at between 5 and $160 \mu \mathrm{mol} / 1$ [52], acyl-CoAs are amphipathic and are probably bound either to cell membranes or acyl-CoA-binding protein; neither their free concentration nor their availability as substrates or inhibitors can be known with certainty.

\section{Conclusion}

Although much has been accomplished, many important questions remain. Future studies are required to determine the subcellular location of each ACSL and ACSVL isoform. Purification and crystallization of mammalian ACSL and ACSVL isoforms is needed to confirm the mechanism of action and discover how these enzymes differ in their affinity for FAs of different chain lengths. Functionally, it will be important to learn the conditions 
under which ACS activity activates AMPK and how the ACSL isoforms can direct their acyl-CoA products toward independent downstream pathways.

\section{Acknowledgments}

This work was supported by NIH grants DK056598, R01-DK59935 and DK59935-S1 (ARRA) (R.A.C.), DK40936, U24-DK59635, a postdoctoral fellowship DK082099 (J.L.F.), and the UNC Clinical Nutrition Research Unit (P30 DK56350). Postdoctoral (L.O.L.) and Predoctoral (J.M.E.) fellowships were from the American Heart Association-Mid-Atlantic Region.

\section{References and recommended reading}

Papers of particular interest, published within the annual period of review, have been highlighted as:

- of special interest

•• of outstanding interest

Additional references related to this topic can also be found in the Current World Literature section in this issue (p. 260).

1. Watkins PA, Maiguel D, Jia Z, Pevsner J. Evidence for 26 distinct acylcoenzyme A synthetase genes in the human genome. J Lipid Res. 2007; 48:2736-2750. [PubMed: 17762044]

2•. Watkins PA. Very-long-chain acyl-CoA synthetases. J Biol Chem. 2008; 283:1773-1777. [PubMed: 18024425] [An excellent summary of the properties of ACSVL isoforms and controversies about them.]

3••. Gulick AM. Conformational dynamics in the Acyl-CoA synthetases, adenylation domains of nonribosomal peptide synthetases, and firefly luciferase. ACS Chem Biol. 2009; 4:811-827. [PubMed: 19610673] [This review of crystallographic and biochemical data on the ANL superfamily covers the pertinent publications from the 1950s to the present. Gulick thoroughly presents the data for the domain alternation catalytic mechanism, in which the enzyme rotates the C-terminal domain 1408 in reference to the active site after the adenylate intermediate is formed before proceeding to the second catalytic step.]

4••. Oba Y, Iida K, Inouye S. Functional conversion of fatty acyl-CoA synthetase to firefly luciferase by site-directed mutagenesis: a key substitution responsible for luminescence activity. FEBS Lett. 2009; 583:2004-2008. [PubMed: 19450587] [This remarkable study shows that a single amino acid change can convert an ACS to a luciferase.]

5•. Kochan G, Pilka ES, von Delft F, et al. Structural snapshots for the conformation-dependent catalysis by human medium-chain acyl-coenzyme A synthe tase ACSM2A. J Mol Biol. 2009; 388:997-1008. [PubMed: 19345228] [This article provides the only data for a mammalian ACS that has been crystallized.]

6. Doege H, Stahl A. Protein-mediated fatty acid uptake: novel insights from in vivo models. Physiology (Bethesda). 2006; 21:259-268. [PubMed: 16868315]

7. Mashek DG, Coleman RA. Cellular fatty acid uptake: the contribution of metabolism. Curr Opin Lipidol. 2006; 17:274-278. [PubMed: 16680032]

8. Schaffer JE, Lodish HF. Expression cloning and characterization of a novel adipocyte long chain fatty acid transport protein. Cell. 1994; 79:427-436. [PubMed: 7954810]

9. Gargiulo CE, Stuhlsatz-Krouper SM, Schaffer JE. Localization of adipocyte long-chain fatty acylCA synthetase at the plasma membrane. J Lipid Res. 1999; 40:881-892. [PubMed: 10224157]

10. Milger K, Herrmann T, Becker C, et al. Cellular uptake of fatty acids driven by the ER-localized acyl-CoA synthetase FATP4. J Cell Sci. 2006; 119:4678-4688. [PubMed: 17062637]

11. Li LO, Mashek DG, An J, et al. Overexpression of rat long chain acyl-CoA synthetase 1 alters fatty acid metabolism in rat primary hepatocytes. J Biol Chem. 2006; 281:37246-37255. [PubMed: 17028193] 
12••. Li LO, Ellis JM, Paich HA, et al. Liver-specific loss of long chain acyl-CoA synthetase-1 decreases triacylglycerol synthesis and beta-oxidation and alters phospholipid fatty acid composition. J Biol Chem. 2009; 284:27816-27826. [PubMed: 19648649] [This is the first report of an ACSL1 knockout mouse model. The minimal phenotype is surprising, but defects are seen in b-oxidation.]

13••. Lobo S, Wiczer BM, Bernlohr DA. Functional analysis of long-chain acyl-CoA synthetase 1 in 3T3-L1 adipocytes. J Biol Chem. 2009; 284:18347-18356. [PubMed: 19429676] [This report knocked down ACSL1 in 3T3-L1 cells. The authors conclude that ACSL1 is required for FA reesterification after lipolysis. No effect was observed for FA uptake.]

14••. Bu SY, Mashek MT, Mashek DG. Suppression of long chain acyl-CoA synthetase 3 decreases hepatic de novo fatty acid synthesis through decreased transcriptional activity. J Biol Chem. 2009; 284:30474-30483. [PubMed: 19737935] [This report shows that, of the ACSL and ACSVL/FATP enzymes tested, only ACSL3 alters the transcriptional activity of ligand-sensitive transcription factors.]

15••. Yao H, Ye J. Long chain acyl-CoA synthetase 3-mediated phosphatidylcholine synthesis is required for assembly of very low density lipoproteins in human hepatoma Huh7 cells. J Biol Chem. 2008; 283:849-854. [PubMed: 18003621] [This article shows that a specific ACSL has a definite function in lipoprotein metabolism.]

16. Mashek DG, McKenzie MA, Van Horn CG, Coleman RA. Rat long chain acyl-CoA synthetase 5 increases fatty acid uptake and partitioning to cellular triacylglycerol in McArdle-RH7777 cells. J Biol Chem. 2006; 281:945-950. [PubMed: 16263710]

17. Zhou Y, Abidi P, Kim A, et al. Transcriptional activation of hepatic ACSL3 and ACSL5 by oncostatin $\mathrm{M}$ reduces hypertriglyceridemia through enhanced beta-oxidation. Arterioscler Thromb Vasc Biol. 2007; 27:2198-2205. [PubMed: 17761945]

18. Souza SC, Muliro KV, Liscum L, et al. Modulation of hormone-sensitive lipase and protein kinase A-mediated lipolysis by perilipin A in an adenoviral reconstituted system. J Biol Chem. 2002; 277:8267-8272. [PubMed: 11751901]

19••. Forner F, Kumar C, Luber CA, et al. Proteome differences between brown and white fat mitochondria reveal specialized metabolic functions. Cell Metab. 2009; 10:324-335. [PubMed: 19808025] [This group used a novel proteomic technique to determine absolute quantities of proteins within insolated mitochondria from adipose tissues and adipocyte cell lines. The protein composition will aid in understanding differences between the different adipose depots and between adipose and adipocyte-like cells.]

20••. Wiczer BM, Bernlohr DA. A novel role for fatty acid transport protein 1 in the regulation of tricarboxylic acid cycle and mitochondrial function in 3T3-L1 adipocytes. J Lipid Res. 2009; 50:2502-2513. [PubMed: 19535819] [Protein-protein interactions within the mitochondria show a novel effect of an ACSVL/FATP that interacts with a TCA enzyme to enhance its activity.]

21 - Sebastian D, Guitart M, Garcia-Martinez C, et al. Novel role of FATP1 in mitochondrial fatty acid oxidation in skeletal muscle cells. J Lipid Res. 2009; 50:1789-1799. [PubMed: 19429947] [This article reports co-immunoprecipitation of overexpressed FATP1 and CPT1 and a synergistic effect of FATP1 and CPT1 on FA oxidation.]

22. Richards MR, Harp JD, Ory DS, Schaffer JE. Fatty acid transport protein 1 and long-chain acyl coenzyme A synthetase 1 interact in adipocytes. J Lipid Res. 2006; 47:665-672. [PubMed: 16357361]

23•. Soupene E, Kuypers FA. Mammalian long-chain acyl-CoA synthetases. Exp Biol Med (Maywood). 2008; 233:507-521. [PubMed: 18375835] [A good summary of ACS variants.]

24. Jia Z, Pei Z, Maiguel D, et al. The fatty acid transport protein (FATP) family: very long chain acylCoA synthetases or solute carriers? J Mol Neurosci. 2007; 33:25-31. [PubMed: 17901542]

25. Starai VJ, Celic I, Cole RN, et al. Sir2-dependent activation of acetyl-CoA synthetase by deacetylation of active lysine. Science. 2002; 298:2390-2392. [PubMed: 12493915]

26. North BJ, Sinclair DA. Sirtuins: a conserved key unlocking AceCS activity. Trends Biochem Sci. 2007; 32:1-4. [PubMed: 17141505]

27••. Schwer B, Eckersdorff M, Li Y, et al. Calorie restriction alters mitochondrial protein acetylation. Aging Cell. 2009; 8:604-606. [PubMed: 19594485] [This study reports the association of a physiologic state with a posttranslational modification.] 
28. Jump DB. Dietary polyunsaturated fatty acids and regulation of gene transcription. Curr Opin Lipidol. 2002; 13:155-164. [PubMed: 11891418]

29. Tomoda H, Igarashi K, Cyong JC, Omura S. Evidence for an essential role of long chain acyl-CoA synthetase in animal cell proliferation. J Biol Chem. 1991; 266:4214-4219. [PubMed: 1999415]

30• Mashima T, Sato S, Okabe S, et al. Acyl-CoA synthetase as a cancer survival factor: its inhibition enhances the efficacy of etoposide. Cancer Sci. 2009; 100:1556-1562. [PubMed: 19459852] [Novel evidence for a causative role for an ACS in cancer.]

31 - Mashima T, Sato S, Sugimoto Y, et al. Promotion of glioma cell survival by acyl- CoA synthetase 5 under extracellular acidosis conditions. Oncogene. 2009; 28:9-19. [PubMed: 18806831] [Novel evidence for a protective role for an ACS in cancer.]

32. Pei Z, Sun P, Huang P, et al. Acyl-CoA synthetase VL3 knockdown inhibits human glioma cell proliferation and tumorigenicity. Cancer Res. 2009; 15:9175-9182. [PubMed: 19920185] [This article associates ACSVL3 with glioma cell proliferation.]

33•. Teng AC, Adamo K, Tesson F, Stewart AF. Functional characterization of a promoter polymorphism that drives ACSL5 gene expression in skeletal muscle and associates with dietinduced weight loss. FASEB J. 2009; 23:1705-1709. [PubMed: 19218499] [This is one of the first studies to identify an ACSL polymorphism with a functional effect on human physiology.]

34•. Perera F, Tang WY, Herbstman J, et al. Relation of DNA methylation of 5'-CpG island of ACSL3 to transplacental exposure to airborne polycyclic aromatic hydrocarbons and childhood asthma. PLoS One. 2009; 4:e4488. [PubMed: 19221603] [This is a curious association of ACSL3 DNA methylation related to asthma. No ACSL was previously thought to affect a specific immunological or pulmonary function.]

35••. Meloni I, Parri V, De Filippis R, et al. The XLMR gene ACSL4 plays a role in dendritic spine architecture. Neuroscience. 2009; 159:657-669. [PubMed: 19166906] [This is the first paper to identify an abnormality possibly related to the brain of patients with ACSL4 X-linked mental retardation.]

36•. Zeman M, Vecka M, Jachymova M, et al. Fatty acid CoA ligase-4 gene polymorphism influences fatty acid metabolism in metabolic syndrome, but not in depression. Tohoku J Exp Med. 2009; 217:287-293. [PubMed: 19346733] [This report links a polymorphism in Acsl4 to persons with the metabolic syndrome.]

37•. Kotronen A, Yki-Jarvinen H, Aminoff A, et al. Genetic variation in the ADIPOR2 gene is associated with liver fat content and its surrogate markers in three independent cohorts. Eur J Endocrinol. 2009; 160:593-602. [PubMed: 19208777] [This report links polymorphisms in Acs14 to human liver and circulating TAG content.]

38•. Klar J, Schweiger M, Zimmerman R, et al. Mutations in the fatty acid transport protein 4 gene cause the ichthyosis prematurity syndrome. Am J Hum Genet. 2009; 85:248-253. [PubMed: 19631310] [This report shows phenotypic similarities between mice and humans with defective FATP4.]

39. Herrmann T, van der Hoeven F, Grone HJ, et al. Mice with targeted disruption of the fatty acid transport protein 4 (Fatp 4, Slc27a4) gene show features of lethal restrictive dermopathy. J Cell Biol. 2003; 161:1105-1115. [PubMed: 12821645]

40. Gonzalez AA, Kumar R, Mulligan JD, et al. Metabolic adaptations to fasting and chronic caloric restriction in heart, muscle, and liver do not include changes in AMPK activity. Am J Physiol Endocrinol Metab. 2004; 287:E1032-E1037. [PubMed: 15251868]

41. Mulligan JD, Gonzalez AA, Stewart AM, et al. Upregulation of AMPK during cold exposure occurs via distinct mechanisms in brown and white adipose tissue of the mouse. J Physiol. 2007; 580:677-684. [PubMed: 17272339]

42••. Gauthier MS, Miyoshi H, Souza SC, et al. AMP-activated protein kinase is activated as a consequence of lipolysis in the adipocyte: potential mechanism and physiological relevance. $\mathrm{J}$ Biol Chem. 2008; 283:16514-16524. [PubMed: 18390901] [An interesting paper showing activation of AMPK related to TAG hydrolysis in fat cells in culture.]

43••. Wiczer BM, Lobo S, Machen GL, et al. FATP1 mediates fatty acid-induced activation of AMPK in 3T3-L1 adipocytes. Biochem Biophys Res Commun. 2009; 387:234-238. [PubMed: 19560442] [This article shows AMPK activation resulting from overexpression of FATP1.] 
44. Clark H, Carling D, Saggerson D. Covalent activation of heart AMP-activated protein kinase in response to physiological concentrations of long-chain fatty acids. Eur J Biochem. 2004;

271:2215-2224. [PubMed: 15153111]

45•. Steinberg GR, Kemp BE. AMPK in health and disease. Physiol Rev. 2009; 89:1025-1078. [PubMed: 19584320] [This is a good basic review of AMPK.]

46. Fediuc S, Gaidhu MP, Ceddia RB. Regulation of AMP-activated protein kinase and acetyl-CoA carboxylase phosphorylation by palmitate in skeletal muscle cells. J Lipid Res. 2006; 47:412-420. [PubMed: 16304351]

47. Maeda K, Cao H, Kono K, et al. Adipocyte/macrophage fatty acid binding proteins control integrated metabolic responses in obesity and diabetes. Cell Metab. 2005; 1:107-119. [PubMed: 16054052]

48. Za'tara G, Bar-Tana J, Kalderon B, et al. AMPK activation by long chain fatty acyl analogs. Biochem Pharmacol. 2008; 76:1263-1275. [PubMed: 18812171] [Another interesting example of AMPK activation related to FA activation.]

49. Watt MJ, Steinberg GR, Chen ZP, et al. Fatty acids stimulate AMP-activated protein kinase and enhance fatty acid oxidation in L6 myotubes. J Physiol. 2006; 574:139-147. [PubMed: 16644805]

50. Taylor EB, Ellingson WJ, Lamb JD, et al. Long-chain acyl-CoA esters inhibit phosphorylation of AMP-activated protein kinase at threonine-172 by LKB1/STRAD/MO25. Am J Physiol Endocrinol Metab. 2005; 288:E1055-E1061. [PubMed: 15644453]

51. Djouder N, Tuerk RD, Suter M, et al. PKA phosphorylates and inactivates AMPKalpha to promote efficient lipolysis. EMBO J. 2009; 29:469-481. [PubMed: 19942859]

52. Færgeman NJ, Knudsen J. Role of long-chain fatty acyl-CoA esters in the regulation of metabolism and in cell signalling. Biochem J. 1997; 323:1-12. [PubMed: 9173866] 


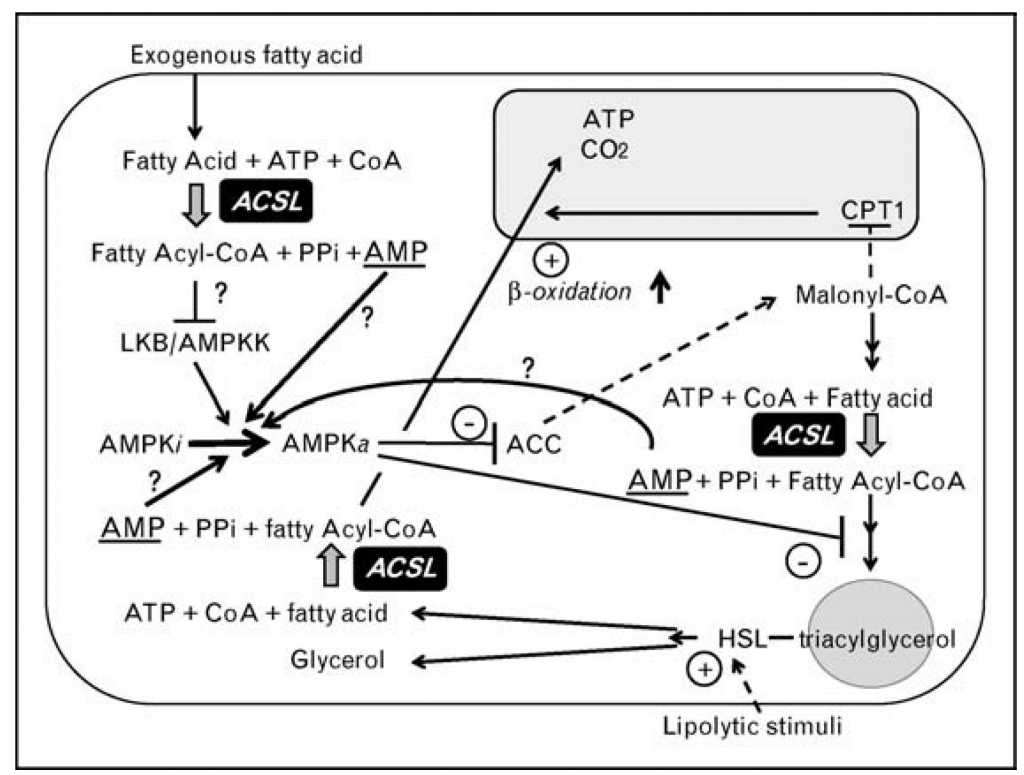

Figure 1. Activation of fatty acids by long-chain acyl-coenzyme A synthetase may activate AMPactivated kinase

When exogenous fatty acids enter the cell, are synthesized de novo, or are hydrolyzed from intracellular triacylglycerol stores, they must first be activated by a long chain acyl-CoA synthetase (ACSL) to form fatty acyl-CoAs. They can then enter downstream metabolic pathways. Because ATP is a substrate for ACSL and AMP is a product, the increased AMP/ATP ratio, a signal of low cellular energy stores, could theoretically activate AMPactivated kinase (AMPK). Activated AMPK inhibits triacylglycerol synthesis and acetylCoA carboxylase (ACC). The resulting decrease in malonyl-CoA levels diminishes fatty acid synthesis and also relieves the inhibition of carnitine palmitoyltransferase-1 (CPT1) by malonyl-CoA, allowing fatty acyl-CoA to be converted to acyl-carnitine and enter the mitochondria for $\beta$-oxidation. Thus, activated AMPK would generate an increase in ATP production. A prolonged lipogenic stimulus [which activates hormone-sensitive lipase (HSL)], may also inactivate AMPK to modulate these effects. It seems unlikely, however, that ACSL would activate AMPK under physiological conditions that stimulate triacylglycerol synthesis. 